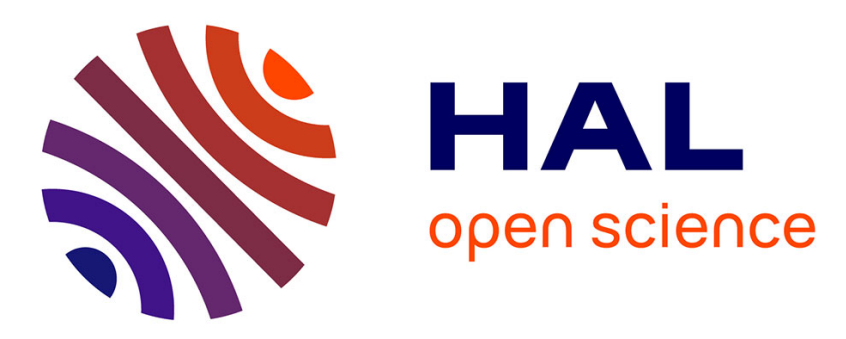

\title{
Depression in Swedish women: relationship to factors at birth
}

\author{
Pia Gudmundsson, Susan Andersson, Deborah Gustafson, Margda Waern, \\ Svante Östling, Tore Hällström, Sigurdur Palsson, Ingmar Skoog, Lena \\ Hulthen
}

\section{To cite this version:}

Pia Gudmundsson, Susan Andersson, Deborah Gustafson, Margda Waern, Svante Östling, et al.. Depression in Swedish women: relationship to factors at birth. European Journal of Epidemiology, 2010, 26 (1), pp.55-60. 10.1007/s10654-010-9508-7 . hal-00625151

\section{HAL Id: hal-00625151 https://hal.science/hal-00625151}

Submitted on 21 Sep 2011

HAL is a multi-disciplinary open access archive for the deposit and dissemination of scientific research documents, whether they are published or not. The documents may come from teaching and research institutions in France or abroad, or from public or private research centers.
L'archive ouverte pluridisciplinaire HAL, est destinée au dépôt et à la diffusion de documents scientifiques de niveau recherche, publiés ou non, émanant des établissements d'enseignement et de recherche français ou étrangers, des laboratoires publics ou privés. 
Gustafson, Deborah

+17182701581

\section{Depression in Swedish Women: Relationship to Factors at Birth}

Pia Gudmundsson ${ }^{1}$, Susan Andersson ${ }^{2,3}$, Deborah Gustafson ${ }^{1,4}$, Margda Waern ${ }^{1}$, Svante Östling ${ }^{1}$, Tore Hällström ${ }^{1,5}$, Sigurdur Palsson ${ }^{1}$, Ingmar Skoog ${ }^{1}$, Lena Hulthen ${ }^{2}$

${ }^{1}$ Institute of Neuroscience and Physiology, Section for Psychiatry and Neurochemistry

Sahlgrenska Academy at University of Gothenburg, Sweden; ${ }^{2}$ Institute of Medicine, Section for

Clinical Nutrition Sahlgrenska Academy at University of Gothenburg, Sweden; ${ }^{3}$ Department of

Epidemiology, Astra Zeneca, AB, Mölndal, Sweden; ${ }^{4}$ SUNY-Downstate Medical Center,

Departments of Neurology and Medicine, New York, USA. ${ }^{5}$ Department of clinical neuroscience, Section for psychiatry/Huddinge, Karolinska Institutet, Stock holm, Sweden.

Address for correspondence and reprint requests:

Deborah R. Gustafson, $\mathrm{PhD}$

SUNY-Downstate Medical Center

Departments of Neurology and Medicine

450 Clarkson Avenue, Box 1213

Brooklyn, New York 11203, USA

Telephone: +1 7182701581

Fax: +1 7182703840

E-mail: deb.gustafson@ neuro.gu.se or deborah.gustafson@downstate.edu 
Abstract Depression is a common and serious disorder that may have developmental origins. Birth-related factors have been related to childhood and adult occurrence of somatic as well as psychiatric disorders, but studies on the relationship between birth-related factors and depression are few and show mixed results. In addition, varying methods have been used to assess depression. Standardized clinical criteria to diagnose depression, combined with birth data collected from midwife records have not been used in most studies. Participants in the Prospective Population Study of Women in Sweden (803 wome n), born 1914, 1918, 1922 and 1930, provide information on birth factors and depression. Women participated from 1968 at mid-life ages of 38-60 years, to 2000, when they were age 78-92 years. Original birth records containing birth weight, length, head circumference, and gestational time, as well as social factors were obtained. Lifetime depression was diagnosed via multiple information sources. Symptoms were assessed using the Comprehensive Psychopathological Rating Scale and diagnoses were based on DSM-III-R criteria. Over their lifetime, $44.6 \%$ of women in this sample experienced depression. Birth weights $\leq 3500$ grams [odds ratio (OR), age-adjusted=1.72; $95 \%$ CI 1.29-2.28, $P<0.001]$ and shorter gestational time (OR, age-adjusted=1.13; 95\% CI 1.04-1.24, $P=0.005)$ were independently associated with a higher odds of lifetime depression in a logistic regression model adjusted for age. Lower than median birth weights and shorter gestational time were related to lifetime depression in women. Both neurodevelopmental and environmental contributions to lifetime depression may be considered.

Keywords Depression, birth factors, birth weight, gestational age, population-based, women 


\section{Introduction}

The fetal origins, or Barker hypothesis, was first described by Barker and Os mond in 1986 [1] and proposes that somatic diseases occurring during adult life, such as coronary heart disease, type 2 diabetes, stroke and hy pertension originate in developmental plasticity, are responses to undernutrition during fetal life and infancy [2]. Evidence accumulates for a fetal origin of psychiatric disorders, as well [3-4]. Historically, birth weight, birth length, head circumference, and gestational time have been common birth parameters measured to better understand potential developmental influence rs of the central ne rvous system. Birth weight represents accumulation of adipose tissue during fetal development, which has been shown to contribute to short- and long-term health [5]. The importance of fetal fat accumulation is illustrated by the observation that pre mature babies are at higher risk of slower neurological development and smaller brain size [6]. Thus, birth weight has been deemed an important marker of neurodevelopment.

Since depression is a common and multifactorial disorder that is episodic over the life course, and often leads to serious consequences such as increased mortality and suicide, [7] it is of great importance to identify early factors that may be related to its occurrence and etiology. Epidemiological studies are valuable to evaluate this question.

Several studies show an association between lower birth weight and later depression, [4, 8-10] while others find no such relationship [11-12]. Few report on both birth weight and gestational time. Among those that do, two found an association for shorter gestational time, [13-14] and 
another supported the finding on lower birth weight [15]. Independent effects of both factors in relationship to depression have not been reported. Most of these studies measure depression at one point in time and focus on younger populations.

We had the opportunity to investigate birth-related factors including birth weight, gestational time, birth length, and head circumference, in relationship to lifetime depression evaluated on multiple occasions using several different sources of information, in a sample of women observed longitudinally over 32 years from mid- to late-life. These women were enrolled in the Prospective Population Study of Women (PPSW) in Gothenburg, Sweden.

\section{Methods}

\section{Subjects}

PPSW began in 1968-69 with a baseline examination of 1381 women (90.6\% participation rate) [16] born in 1914, 1918, 1922 and 1930, who originated from a representative sample obtained from the Revenue Office Register in Gothenburg. Of these women, 1140 participated in at least one psychiatric examination, and for 803 of them birth factor information, retrieved from original midwife records in the 1990 's, was available. Participants were re-examined over the next 32 years in 1974, 1980, 1992, and 2000. Informed consent was obtained from all participants and/or their relatives. The study was approved by the Ethics Committee for Medical Research of University of Gothenburg.

\section{A comparison of women with versus without birth factor information in the 1968 baseline} sample showed that women with birth factor information we re younger in 1968 compared 


\section{to those without $(45.6(5.8)$ versus $46.8(5.3)$ years, $p<0.001)$, however there was no difference in level of education obtained in adult life (OR 1.21 95\% CI 0.96, 1.53, p=0.113).}

\section{Birth-related factors}

Birth-related factors were abstracted from original midwife records as previously described [17]. Biological birth-related factors that were recorded include birth weight, gestational time, birth length, and head circumference. While information on birth weight and gestational time were routinely recorded for all birth cohorts studied, birth length was first recorded in Swedish midwife records in 1917, and head circumference in 1926. Additional information was collected when available, including parental social group (categorized in five class levels based on occupation of the father, or if missing or no father, on the mother), maternal age, maternal parity and the participant's birth location (i.e., home vs. hospital) and twin status [17].

\section{Psychiatric examination}

Psychiatric examinations were performed by psychiatrists in 1968, 1974, 1980, and 1992 and by psychiatric nurses in 2000. Beginning in 1987, the diagnosis of depression was made using criteria described in the Diagnostic and Statistical Manual of Mental Disorders, 3rd edition revised (DSM-III-R) [18], and also retrospectively for examinations in 1968, 1974 and 1980. Application of these criteria was based on the psychiatric examinations, history of previous depression as evaluated by a clinical psychiatrist [19,20], and depression between examinations, information from medical records and the Swedish Hospital Discharge Registry. At the psychiatric examinations, the Comprehensive Psychopathological Rating Scale (CPRS) [21] was used to rate psychiatric symptoms and signs at face-to-face examinations. Medical records were collected from hospitals and outpatient departments including psychiatric and primary care in 
Gothenburg. The Swedish Hospital Discharge Registry provided diagnostic information for individuals discharged from hospitals since 1978. Lifetime depression included major depressive disorder, dysthymia, and depression not otherwise specified. A diagnosis of dysthymia was made without the two year criterion as information on symptom duration was not specified [22].

\section{Statis tical analyses}

Birth weights were available for all 803 participants; however birth record information was incomplete for other birth-related factors. Gestational time was available for 750, birth length for 648, and head circumference for 444 women. Only values within acceptable ranges were used. All values for birth weight, birth length, and head circumference fulfilled this criterion. However, due to some extreme outliers for gestational time, only individuals with gestational times ranging 1.5 standard deviations from the mean of 40 weeks (between $35.2-44.9$ weeks) $(n=715)$ were included in analyses with gestational time. Varying sample sizes are represented across analyses due to the varying completeness of birth factor information. Birth weight, gestational time,

\section{birth length, and head circumfe rence we re normally distributed.}

Means and standard deviations were calculated for all continuous variables, and t-tests were used to asses s mean differences by depression status. Continuous measures of birth factors were used in all initial analyses, and further analyses were conducted considering birth weight, birth length, and head circumference in quintiles and as dichotomous variables using median values as cutoffs. Birth weight was considered as a continuous variable in 500 gram increments. Pearson correlation coefficients were calculated for all continuous variables. Partial correlation coefficients were calculated after adjustment for age. Linear trends in means of birth factors across level of social group were also tested. Due to inaccuracies in estimating date of depression onset, logistic regression analyses were used to estimate the odds of lifetime depression by birth- 
related factors, both biological and social. Age-adjusted birth-related factors that were significant at a level of $P<0.05$, were subsequently included in multivariate models. Cox proportional hazards analyses were run to estimate the relationship between birth factors and overall mortality. Results were considered statistically significant at $P<0.05$. SPSS, version 15.0 was used to analyze study data.

\section{Results}

Over their lifetime, $44.6 \%$ of women in this sample experienced depression. Their average birth parameters are shown in table 1. Evaluating interrelationships among birth-related factors showed that birth weight was associated with a variety of other factors. Higher maternal age (Pearson's partial $r$, cohort adjusted $=0.135, \mathrm{df}=799, P<0.001)$ and maternal parity (Pearson's partial $r$, cohort adjusted $=0.253, \mathrm{df}=800, P<0.001)$ were related to a higher birth weight. Increasing level of parental social group was related to higher birth weight $(P$ for trend $=0.032)$. Babies born at home (observed among $37.1 \%$ ) were heavier (mean (SD)=3562 (559) vs 3482 (558) grams, ageadjusted $P=0.049$ ), and being one of a twin pair (observed among $2.1 \%$ ) was associated with a lower mean birth weight (mean $(\mathrm{SD})=2707$ (519) vs $3529(548)$, age-adjusted $P<0.001)$ and a shorter gestational time (mean $(\mathrm{SD})=38.5(2.2)$ vs $40.1(1.7)$, age-adjusted $P=0.001)$ than being a non-twin. As expected, birth-related factors were correlated with each other (range of ageadjusted Pearson's partial $r=0.210-0.694, \mathrm{df}=416$ ).

Overall, lower average birth weight and shorter gestational time were related to lifetime depression (table 2). Further analyses showed that birth weights of 3500 grams or less (3500 grams was the median and approximate mean of the sample), and shorter gestational time were 
independently associated with higher odds of lifetime depression (table 3). Dose-response relationships were not observed. There were no differences between women with and without lifetime depression regarding other biological birth-related factors including birth length and head circumference. Birth-related factors were not related to overall mortality (data not shown).

(Table 1 here)

(Table 2 here)

(Table 3 here)

As expected, individuals below the median birth weight had shorter mean gestational time compared to those with birth weight above the median (39.9 (1.8) vs 40.4 (1.6) weeks, $P<0.001)$. The direction of the relationship between gestational time and lifetime depression did not differ by median birth weight strata. Among those with a birth weight greater than the median, shorter gestational time was related to lifetime depression (OR 1.23, 95\% CI 1.06-1.43, $P=0.006)$.

However, there was no evidence of an interaction between birth weight and gestational time in a logistic regression model.

\section{Discussion}

To our knowledge, this study is the first to show independent associations between both birth weight and gestational time and a lifetime diagnosis of depression. This study supports previously observed associations between lower birth weight $[4,8-10,15]$ or shorter gestational time [13-14] and higher occurrence of depression. However this is the first to report independent effects of both of these birth factors in the same sample. 
Our re ported prevalence of lifetime depression is supported by other studies [23-25]. Some studies report lower prevalences of lifetime depression but these studies are often retrospective and do not include elderly populations [23]. It has been suggested that lifetime prevalence of mental disorders may be twice as high in prospective versus retrospective studies [26]. Our group reported a comparable incidence of depression in adults age 70 years and older [25].

Important strengths of this epidemiological investigation is that it includes: 1) a representative and well characterized population sample; 2) birth data that were abstracted from original midwife records, which are more accurate than self-report [27]; 3) rigorous standardized criteria for diagnosing clinical psychiatric disorders; 4) depression ascertained over more than 32 years of observation and based on multiple sources of information; and 5) independent collection of birth factor information and psychiatric endpoints, thus reducing systematic bias. Finally, there was consideration for several potential confounding factors including maternal age, maternal parity, parental social group, birth location, and twin status.

Differences in results obtained from epidemiological studies evaluating birth factors in relationship to mental disorders may be due to a variety of factors. Not all studies have all birth parameters, birth data are of varying quality, and criteria used to diagnose depression vary. To our knowledge we are one of the only studies to have a life time diagnosis of depression in addition to it being based on clinical DSM-III-R criteria.

\section{Possible etiological mechanis ms}


The developmental origins hypothesis, simply described, is the influence of early life environment on structural and functional development during critical periods, which may lead to short- and long-term consequences for the individual, even later disease [2]. In relationship to depression, lower birth weight and shorter gestational time may be related to glucocorticoidinduced programming of the hypothalamic-pituitary-adrenal (HPA) axis. In addition, low birth weight has been as sociated with higher plasma cortisol concentrations in adults [28] and elevated cortisol has been related to depression in adults [29].

Another potential link between birth weight, gestational time, and depression is related to adipose tissue. Birth weight is, in part, a marker of adipose tissue stored in the fetus, especially during later development when grams of fetal body fat double between 35 and 40 weeks of gestation [30]. Fetal fat accumulation has long been known to be important for the brain, as premature babies are at higher risk of slower neurological development [6]. Adipose tissue is an important energy source, a source of bioactive free fatty acids, and is also the largest endocrine organ in the human body [30]. Additional biological candidate mechanisms that relate birth weight and gestational time to depression may be related to other hypothalamic-pituitary-end organ axes, such as the hypothalamic-pituitary-thyroid (HPT), [31] and-gonadal (HPG) [32] axes.

Maternal factors during pregnancy may also be related to lower birth weight, shorter gestational time and depression symptoms in the offspring. Some of these factors include external stress, smoking, psychiatric illness, preeclampsia, SES, and maternal birth weight [33]. Some of these factors are available in this study, but we did not have the power to investigate them. 
One may suspect that the serious outcomes associated with lower birth weights are related to severe intrauterine growth retardation and pathologically low birth weights. However, our results regarding birth weights of 3500 grams or less, are in line with two other studies. One showed that women weighing less than 3000 grams at birth had an increased risk of depression later on, in early adulthood [9]; and another showed that participants with birth weights less than $2500 \mathrm{~g}$ had an increased risk of major depression compared to those weighing more than $3500 \mathrm{~g}$ [4].

Processes traditionally associated with pathologically low birth weights and preterm births occur over a continuum, and thus variation within the normal range may confer some degree of susceptibility to developing depression later in life. Lower birth weight and shorter gestational time could cause less neuroplasticity [34] from birth in these women, thus be markers of a low tolerance or susceptibility state for depression.

As with all research studies, there are limitations. First, all members of the sample are female, White, and living in Sweden, thereby limiting sex, racial, and cultural generalizability. However the concurrence of our findings with other birth cohort studies is encouraging. Second, the sample size is small compared to other developmental origins study cohorts. However, our rigorously collected exposure and outcomes data and long follow-up time fill a niche in the developmental origins cohort literature. Third, gestational time is estimated based on date of last menstrual period. Given the socioeconomic and nutritional conditions during the early twentieth century, leading to more prevalent amenorrhea and dys menorrhea, as well as lack of birth control leading to rapidly occurring consecutive births, the dates for this parameter may have been imprecise. However this is one of the methods still used today; and we reduced the influence of potential outliers by including only individuals with gestational times ranging 1.5 standard deviations from the mean. Fourth, depression is an episodic disorder. 
Despite the use of case records, registry data and retrospective information to complement depression diagnoses via psychiatric examinations over a 32 year period, there are most likely cases of depression in the non-depressed group. Fifth, a limited number of confounders are available for conside ration in these analyses, making it difficult to tease out the relative contribution of potentially etiological factors. The potential for residual confounding is obvious, and something that cannot be overcome given the historical nature of the birth factors data. We are using birth factors data attained via secondary data collection efforts more than $\mathbf{7 0}$ years after they were originally recorded. These data are not typically available in population-based studies of adults, and are strengths of this dataset. Even so, there are many unknowns related to maternal and paternal exposures, fetal development, and post-natal development. These factors are beyond the scope of this paper. Finally, history of previous depression prior to the baseline examination in 1968 may be subject to recall bias, and was not ascertained in the structured fashion that was employed during the observation period that followed. However, if we excluded these women from the analyses, results were similar in all essential aspects, although not statistically significant due to a reduction in power. In conclusion, lower weight at birth and shorter gestational time were related to lifetime depression in women, which suggests a neurodevelopmental etiology of depression. 


\section{Acknowledgement}

\section{Funding for birth factors collection}

This work was supported by grants from the Swedish Research Council [grant number B96-27X11659, B96-19X-07509]; The Swedish Society of Medicine; The Swedish Nutrition Foundation; The Faculty of Medicine Göteborg University; Sahlgresnka University Hospital Research Fund; the Wilhelm and Martina Lundgren Foundation; and the Leon Memorial Fund.

\section{Funding for longitudinal study and analyses}

This work was supported by grants from the Swedish Research Council [grant number 20058460, 11 337, 11 267, 825-2007-7462); the Swed ish Council for Working Life and Social Research [grant number 2001-2835, 2646, 2003-0234, 2004-0145, 0150, 2006-0020, 0596, 1506, 2008-1229, 1111]; Stiftelsen Söderström-Königska Sjukhemmet; Stiftelsen för Gamla Tjänarinnor; Handlanden Hjalmar Svenssons Forskningsfond; Stiftelsen Professor Bror Gadelius' Minnesfond; The Alzheimer's Association Stephanie B. Overstreet Scholars (IIRG-00-2159); The Alzheimer's Association Zenith Award (ZEN-01-3151); The Göteborg Medical Society; The Bank of Sweden Tercentenary Foundation Alma och Anna Yhlen's Foundation; the Göteborg Medical Services and Social Services Administrations; the Fredrik and Rosa von Malmborgs Foundation for Brain Research; EU FP7 project LipiDiDiet, Grant Agreement N² 211696; and the Swedish Brain Power Project.

\section{Ethical approval}

The study was approved by the Ethics Committee for Medical Research of University of Gothenburg. 


\section{References}

1. Barker DJ, Osmond C. Infant mortality, childhood nutrition, and ischaemic heart disease in England and Wales. Lancet. 1986; (8489): 1077-81.

2. Barker DJ. The developmental origins of adult disease. Eur J Epidemiol. 2003; 18: 733-6.

3. Brown AS, van Os J, Driessens C, Hoek HW, Susser ES. Further evidence of relation between prenatal famine and major affective disorder. Am J Psychiatry. 2000; 157: 190-5.

4. Nomura Y, Wickramaratne PJ, Pilowsky DJ, et al. Low birth weight and risk of affective disorders and selected medical illness in offspring at high and low risk for depression. Compr Psychiatry. 2007; 48: 470-8.

5. Kind KL, Moore VM, Davies MJ. Diet around conception and during pregnancy - effects on fetal and neonatal outcomes. Reprod Biomed, online. 2006; 12: 532-541.

6. Medina JM, Tabernero A. Lactate utilization by brain cells and its role in CNS development. J Neurosci Res. 2005; 79: 2-10.

7. Palsson S, Skoog I. The epidemiology of affective disorders in the elderly: a review. Int Clin Psychopharmacol. 1997; 12 (Supp17): s3-13.

8. Thompson C, Syddall H, Rodin I, Osmond C, Barker DJ. Birth weight and the risk of depressive disorder in late life. Br J Psychiatry. 2001; 179: 450-5.

9. Gale CR, Martyn CN. Birth weight and later risk of depression in a national birth cohort. $\mathrm{Br} \mathbf{J}$ Psychiatry. 2004; 184: 28-33. 
10. Costello EJ, Worthman C, Erkanli A, Angold A. Prediction from low birth weight to female adolescent depression. Arch Gen Psychiatry. 2007; 64: 338-44.

11. Osler M, Nordentoft M, Andersen AM. Birth dimensions and risk of depression in adulthood: cohort study of Danish men born in 1953. Br J Psychiatry. 2005; 186: 400-3.

12. Vasiliad is HM, Gilman SE, Buka SL. Fetal growth restriction and the development of major depression. Acta Psychiatr Scand. 2008; 117:306-12.

13. Raikkonen K, Pesonen AK, Kajantie E, et al. Length of gestation and depressive symptoms at age 60 years. Br J Psychiatry. 2007; 190: 469-74.

14. Inskip HM, Dunn N, Godfrey KM, Cooper C, Kendrick T. Is birth weight associated with risk of depressive symptoms in young women? Evidence from the Southampton Women's Survey. Am J Epidemiol. 2008; 167: 164-8.

15. Mallen C, Mottram S, Thomas E. Birth factors and common mental health problems in young adults: a population-based study in North Staffordshire. Soc Psychiatry Psychiatr Epidemiol. 2008; 43: 325-30.

16. Bengtsson C, Blohme G, Hallberg L, et al. The study of women in Gothenburg 1968-1969-a population study. General design, purpose and sampling results. Acta Med Scand. 1973; 193:311-8.

17. Andersson SW, Niklasson A, Lapidus L, Hallberg L, Bengtsson C, Hulthen L. Sociodemographic characteristics influencing birth outcome in Sweden, 1908-1930. Birth 
variables in the Population Study of Women in Gothenburg. J Epidemiol Community Health. 2000; 54: 269-78.

18. APA. Diagnostic and statistical manual of mental disorders, third edition - revised (DSMIII-R). Washington, D.C., U.S.A. American Psychiatric Association 1987.

19. Hallstrom T, Persson G. The relationship of social setting to major depression. Acta Psychiatr Scand. 1984; 70: 327-336.

20. Hallstrom T. The relationships of childhood socio-demographic factors and early parental loss to major depression in adult life. Acta Psychiatr Scand. 1987; 75: 212-216.

21. Asberg M, Montgomery SA, Perris C, Schalling D, Sedvall G. A comprehensive psychopathological rating scale. Acta Psychiatr Scand. 1978; (Suppl): 5-27.

22. Skoog I, Nilsson L, Landahl S, Steen B. Mental disorders and the use of psychotropic drugs in an 85-year-old urban population. Int Psychogeriatr. 1993; 5: 33-48.

23. Andrews G, Poulton R, Skoog I. Lifetime risk of depression: restricted to a minority or waiting for most? Br J Psych. 2005; 187: 495-496.

24. Jaffee SR, Moffitt TE, Caspi A, Fombonne E, Poulton R, Martin J. Differences in early childhood risk factors for juvenile-onset and adult-onset depression. Arch Gen Psychiatry. $2001 ; 3: 215-222$.

25. Pálsson SP, Östling S, Skoog I. The incidence of first-onset depression in a population followed from the age of 70 to 85 . Psychol Med. 2001; 31: 1159-1168. 
26. Moffitt TE, Caspi A, Taylor A, Kokaua J, Milne BJ, Polanczyk G, Poulton R. How common are common mental disorders? Evidence that lifetime prevalence rates are doubled by prospective versus retrospective ascertainment. Psychol Med. 2010; 6: 899-909.

27. Andersson SW, Niklasson A, Lapidus L, Hallberg L, Bengtsson C, Hulthen L. Poor agreement between self-reported birth weight and birth weight from original records in adult women. Am J Epidemiol. 2000;152:609-16.

28. Phillips DI, Barker DJ, Fall CH, et al. Elevated plasma cortisol concentrations: a link between low birth weight and the insulin resistance syndrome? J Clin Endocrinol Metab. 1998; 83: 757-60.

29. Harris TO, Borsanyi S, Messari S, et al. Morning cortisol as a risk factor for subsequent major depressive disorder in adult women. Br J Psychiatry. 2000; 177: 505-10.

30. Cunnane SC, Crawford MA. Survival of the fattest: fat babies were the key to evolution of the large human brain. Comp Biochem Physiol A Mol Integr Physiol. 2003; 136: 17-26.

31. Kirkegaard C, Faber J. The role of thyroid hormones in depression. Eur J Endocrinol. 1998; 138: $1-9$.

32. Young E, Korszun A. Psychoneuroendocrinology of depression. Hypothalamic-pituitarygonadal axis. Psychiatr Clin North Am. 1998; 21: 309-23.

33. Gustafson D. A life course of adiposity and dementia. Eur J Pharm. 2008; 585: 163-175.

34. Pittenger C, Duman RS. Stress, depression, and neuroplasticity: A convergence of mechanisms. Neuropsychopharmacol. 2008; 33: 88-109. 
Table 1 Birth cohort and birth-related factors in 803 participants in the Prospective

Population Study of Women

\begin{tabular}{lcc}
\hline Birth Cohort & $N(\%)$ & \\
& $89(11.1)$ & \\
1914 & $200(24.9)$ & \\
1918 & $257(32.0)$ & \\
1922 & $257(32.0)$ & \\
1930 & & \\
& & \\
Birth-related Factors* & Mean (SD) & Range \\
& $3511.5(559.6)$ & $1600.0-5500.0$ \\
Weight, grams & $40.1(1.7)$ & $35.3-44.9$ \\
Gestational time, weeks** & $50.2(2.7)$ & $35.0-64.0$ \\
Length, cm & $34.8(1.7)$ & $24.0-40.0$ \\
Head circumference, cm & & \\
\hline
\end{tabular}

* $n=803$ with information on birth weight, 715 with gestational time, 648 with length, and 444 with head circumference

** Gestational time within 1.5 SDs was used, thus included are those greater than 35.2 and less than 44.9 weeks 
Table 2 Birth-related factors in women with versus without lifetime depression

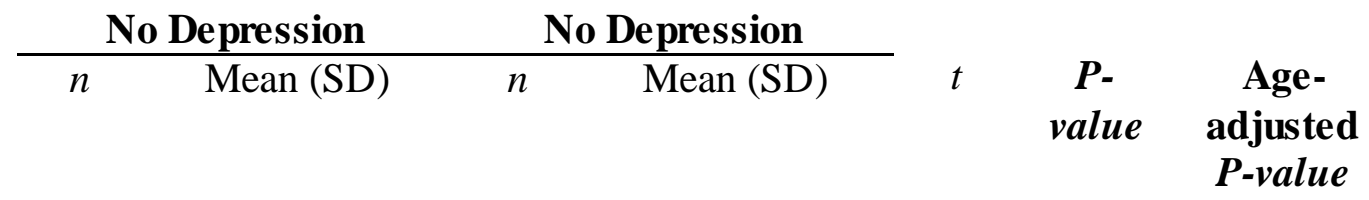

\section{Birth-related Factors*}

\begin{tabular}{lccccccc}
\hline Weight, grams & 445 & $3552.2(555.7)$ & 358 & $3460.9(561.0)$ & 2.3 & 0.021 & 0.025 \\
Gestational time, weeks** & 393 & $40.3(1.6)$ & 322 & $39.9(1.8)$ & 2.9 & 0.004 & 0.005 \\
Length, cm & 360 & $50.3(2.7)$ & 288 & $50.1(2.7)$ & 1.2 & 0.218 & 0.353 \\
Head circumference, cm & 256 & $34.8(1.7)$ & 188 & $34.8(1.7)$ & -0.3 & 0.728 & 0.518 \\
\end{tabular}

* $n=803$ with information on birth weight, 715 with gestational time, 648 with length, and 444 with head circumference

** Gestational time within 1.5 SDs was used, thus included are those greater than 35.2 and less than 44.9 weeks. 
Table 3 Odds of lifetime depression in a population sample of women by birth-related factors, adjusted for birth cohort

OR (95\% CI) Age-adjusted

$P$-value

Birth-Related Factors

\section{Univariate Models}

Biological Factors*

Birth weight $\leq 3500$, grams

$1.72(1.29-2.28) \quad<0.001$

Shorter gestational time, weeks

$1.13(1.04-1.24) \quad 0.005$

Birth length, $\mathrm{cm}$

$0.97(0.92-1.03) \quad 0.353$

Head circumference, $\mathrm{cm}$

$0.96(0.86-1.08) \quad 0.518$

Being a twin

$1.74(0.65-4.66) \quad 0.268$

\section{Social Factors**}

Maternal age, years

$0.99(0.97-1.02) \quad 0.568$

Maternal parity, number of live births

$0.98(0.93-1.04) \quad 0.565$

Increasing parental social group

$0.97(0.86-1.09) \quad 0.570$

Birth location, home versus hospital

$0.96(0.72-1.28)$

0.779

\section{Multivariate Model****}

Birth weight $\leq 3500$, grams

$1.63(1.20-2.22) \quad 0.002$

Gestational time, weeks

$1.11(1.01-1.22) \quad 0.018$

$* \overline{n=803 \text { with information on birth weight, } 715 \text { with ge stational time, } 648 \text { with length, } 444}$ with head circumference, and 803 with twin status

** $n=802$ with information on maternal age, 803 with maternal parity, 797 with parental social group, and 803 with birth location

*** Multivariate model based on factors significant at $P<0.05$, birth weight and gestational time, in cohort-adjusted analyses, $n=715$ 